\title{
Análisis de los resultados de las pruebas estandarizadas Saber Pro en profesionales de la educación
}

Analysis of the results obtained by undergraduate education students in Saber Pro standardized tests

\author{
José Eriberto Cifuentes Medina' \\ joseeriberto.cifuentes@uptc.edu.co \\ José Antonio Chacón Benavides' \\ jose.chacon@uptc.edu.co \\ Israel Alfonso Moreno Pinzón' \\ israel.moreno@uptc.edu.co
}

\section{https://doi.org/10.22209/rhs.v6n2a02}

Recibido: agosto 16 de 2018.

Aceptado: diciembre 13 de 2018.

\section{Resumen}

La investigación se centra en el análisis de los resultados de las pruebas estandarizadas de Colombia Saber Pro, que presentan los estudiantes de la Licenciatura en Educación Básica como requisito de grado. Se descargaron bases de datos de los resultados de las pruebas, desde la página web www.icfesinteractivo. gov.co y se operó con los puntajes numéricos de los estudiantes del programa de Licenciatura en Educación Básica, que se ofrece en modalidad a distancia. Se evidencia que los estudiantes que presentaron las pruebas Saber Pro en el año 2013 fueron objeto de evaluación homogénea y estándar en lo siguiente: Competencias

1 Universidad Pedagógica y Tecnológica de Colombia.
Ciudadanas, Comunicación Escrita, Inglés, Lectura Crítica, Razonamiento Cuantitativo, demostrando que en algunas competencias y componentes se encuentran por debajo del grupo nacional. Las pruebas Saber Pro no son objeto de compromiso serio por parte de los estudiantes, al ser consideradas como un requisito de grado y no como una posibilidad de mejoramiento en la calidad de la educación superior. Se considera que el examen va por un lado y por otro diferente van los estudiantes y la educación sin la remota posibilidad de llegar un punto de convergencia.

Palabras clave: Colombia; Competencias; Licenciatura en Educación Básica; Políticas en Educación; Pruebas Estandarizadas; Saber Pro.

\section{Abstract}

This paper analyses the results obtained by students of a bachelor's degree in primary education in Saber Pro standardized tests in Colombia, which were taken as a graduation requirement. The data bases containing the results of the tests were downloaded from the website www.icfesinteractivo.gov.co and scores obtained by students of the bachelor's degree in primary education (distance mode) were used. The students who took the Saber Pro tests in 2013 were homogeneously and standardly assessed in the following: citizen competences, written communication, English, critical 
reading, quantitative reasoning. The results show that for some competences the students' scores are below the national group. Generally, undergraduate students are not seriously committed to Saber Pro tests, as these are considered as a mere graduation requirement rather than a possibility to improve the quality of higher education. The test is considered to go on one direction while students and education move to another direction, without the slightest possibility of reaching a point of convergence.

Keywords: Colombia, competences, bachelor's degree in primary education, education policies, standardized tests, Saber Pro

\section{Introducción}

I a investigación conlleva, al análisis de las competencias genéricas, específicas, niveles de desempeño y quintiles de cada una de las pruebas presentadas. En el análisis de la información se muestran los porcentajes acumulados de los estudiantes en cada una de las pruebas tanto genéricas como específicas para determinar la concentración de estudiantes con bajos y mayores desempeños en cada una de las pruebas. De igual manera, se presentan gráficos comparativos entre los resultados obtenidos por los estudiantes del programa, con relación a los del grupo de referencia y nacional, en la prueba de competencias genéricas y específicas propias del docente como lo es evaluar, enseñar y formar.

Asimismo, se muestra un análisis descriptivo de los niveles de desempeño de la prueba genérica y de competencias específicas, lo que permite una fácil interpretación de los resultados obtenidos por los estudiantes del programa. Este estudio promueve una reflexión al interior del claustro docente con miras a proponer estrategias fehacientes y efectivas en torno a la preparación de los estudiantes, así como a la autorreflexión de la praxis del docente en los diferentes escenarios del proceso formativo y a la retroalimentación permanente del currículo como eje fundamental del quehacer de la docencia, la investigación y extensión del docente universitario.

Sin embargo, la calidad de los profesionales de la educación no depende únicamente de mejorar los resultados en las pruebas Saber, sino también de diseñar unos adecuados planes de estudio que vayan en función de unas políticas de educación acordes con las necesidades del país; en ello las facultades de educación juegan un papel importante, ya que son las llamadas a plantear planes curriculares acordes con los contextos y escenarios colombianos.

\section{Referentes teóricos}

Entre las consideraciones teóricas se infiere la importancia de las evaluaciones estandarizadas en América Latina, las pruebas internacionales Programme for International Student Assessment (PISA) y Trends in International Mathematics and Science Study (TIMSS), entre otras, y las pruebas nacionales en los diferentes niveles del sistema educativo y su incidencia en la calidad educativa. 
Importancia de las evaluaciones nacionales de logros educativos y la situación de la evaluación estandarizada en América Latina

Se hace necesario resaltar la importancia de las evaluaciones que se desarrollan en cada país de América Latina; es decir, las evaluaciones nacionales, puesto que cobran importancia por su aplicación en contexto y que difieren de las internacionales. Los dos tipos de pruebas miden, comparan y estandarizan, sin embargo, se puede asumir que la evaluación puede servir como base para adoptar políticas educativas mejor fundamentadas, para optimizar la gestión de los sistemas educativos, incluso, como instrumento para la colaboración y el aprendizaje continuo en los mismos. Afirman Ravela \& Arregui et al. (2008) que,

La realización de evaluaciones estandarizadas como forma de conocer mejor la dinámica de procesos y resultados en los sistemas educativos es cada vez más frecuente a nivel regional y mundial, en países de muy diversas culturas y orientaciones ideológicas de gobierno. Prueba de ello es la creciente participación de los países en las evaluaciones internacionales como PISA, TIMSS y PIRLS, y regionales como SERCE (en América Latina) y SACMEQ (en África), así como el desarrollo de diferentes tipos de sistemas nacionales y subnacionales de evaluación (p. 6).

El estudio objeto de estudio, según Ravela \& Arregui et al. (2008), se centra en «evaluaciones de aprendizaje -definido como el cambio ocurrido en los conocimientos y capacidades de cada estudiante a lo largo del año lectivo- y/o de logro educativo, entendido como la acumulación de conocimientos y capacidades a lo largo de toda la vida del estudiante» (p. 5).

Son los organismos multilaterales sin lugar a duda los que promueven las pruebas estandarizadas y según Guevara (2017), señala que:

Las ideas de la UNESCO (Organización de las Naciones Unidas para la Educación, la Ciencia y la Cultura), la OREALC (Oficina Regional de Educación para América Latina y el Caribe), la OEI (Organización de Estados Iberoamericanos) y la CEPAL (Comisión Económica para América Latina y el Caribe) sobre calidad educativa implican las siguientes dimensiones técnicas a través del tiempo, las cuales se unen en una lógica de rendición de cuentas y de medición del rendimiento de los estudiantes (p. 162).

En la perspectiva de Gómez (2004):

Los esfuerzos para conocer el nivel de rendimiento de los estudiantes también han trascendido fronteras. Varios países de la región han competido en pruebas internacionales, como el International Mathematics and Science Study (TIMSS), entre 1994 y 1995, y en 1997 en los exámenes de lenguaje y matemáticas administrados por el Laboratorio Latinoamericano para la Evaluación de la Calidad Educativa de la UNESCO, en la que participaron trece países. Empero, más de dos décadas de esfuerzos para medir el rendimiento escolar y la calidad educativa parecen no arrojar los resultados esperados. Esto se observa en las desesperanzadoras conclusiones de investigadores y autoridades educativas (p. 77).

Y en Colombia no es la excepción, por ello se pretende evidenciar la importancia de las evaluaciones nacionales de orden homogéneo 
como se refiere a las Pruebas Saber $3^{\circ}, 5^{\circ}, 9^{\circ}$, Saber $11^{\circ}$, Saber $\mathrm{T}$ y T y Saber Pro en relación a los propósitos de mejorar por parte del gobierno la cobertura y la calidad de educación y por parte de los organismos multilaterales medir con la misma posibilidad a todos los países aliados de los mismos procesos macroeconómicos para regular a través de las políticas internacionales el desarrollo educativo y social (Tabla 1).

\section{Tabla 1. Importancia de las evaluaciones nacionales.}

\begin{tabular}{|c|c|}
\hline Propósitos & Breve descripción \\
\hline $\begin{array}{l}\text { La evaluación estandarizada ayuda a } \\
\text { visualizar los resultados educativos del } \\
\text { conjunto de estudiantes }\end{array}$ & $\begin{array}{l}\text { La educación es una actividad «opaca», en el sentido } \\
\text { de que sus resultados no son directa ni inmediatamen- } \\
\text { te observables. }\end{array}$ \\
\hline $\begin{array}{l}\text { Las evaluaciones entregan información } \\
\text { sobre el real acceso al conocimiento y a } \\
\text { las capacidades que alcanzan los alum- } \\
\text { nos, más allá de la cantidad de años de } \\
\text { estudio que tengan }\end{array}$ & $\begin{array}{l}\text { En décadas pasadas, la equivalencia entre permanen- } \\
\text { cia en el sistema educativo y acceso al conocimiento y } \\
\text { a las capacidades simbólicas se daba por descontada } \\
\text { y los indicadores para valorar a los sistemas educa- } \\
\text { tivos estaban relacionados con el acceso (matrícula, } \\
\text { cobertura, retención, etc.). }\end{array}$ \\
\hline $\begin{array}{l}\text { Un sistema de evaluación de aprendiza- } \\
\text { jes y/o logros educativos puede aportar } \\
\text { información importante a diversos acto- } \\
\text { res sociales }\end{array}$ & $\begin{array}{l}\text { En la medida que el sistema produzca y comunique } \\
\text { adecuadamente información sobre los aspectos antes } \\
\text { indicados, puede ser un instrumento clave de mejora, } \\
\text { enriqueciendo la comprensión de la situación educati- } \\
\text { va y la toma de decisiones en diversos ámbitos. }\end{array}$ \\
\hline $\begin{array}{l}\text { El desarrollo de un sistema nacional de } \\
\text { evaluación estandarizada puede obligar } \\
\text { a realizar una discusión informada sobre } \\
\text { qué aspectos del currículo formal son } \\
\text { exigibles a todos los estudiantes y a de- } \\
\text { finir con claridad qué es lo que todos los } \\
\text { estudiantes deberían haber aprendido al } \\
\text { final de cada ciclo educativo }\end{array}$ & $\begin{array}{l}\text { La mayoría de los currículos en la región están cons- } \\
\text { tituidos por largas listas de objetivos y temas, todos } \\
\text { deseables, pero no todos realizables. La elaboración } \\
\text { de pruebas nacionales obliga a definir qué es lo que } \\
\text { debe ser considerado como fundamental y, por tanto, } \\
\text { lo que todos los estudiantes deben saber y ser capaces } \\
\text { de hacer. Se pueden usar distintos términos para de- } \\
\text { signar a estas definiciones: estándares, competencias } \\
\text { fundamentales, indicadores de logro, niveles de des- } \\
\text { empeño, metas de aprendizaje, criterios de suficiencia, } \\
\text { entre otros. }\end{array}$ \\
\hline
\end{tabular}

Fuente: elaboración de los autores con información recabada de Ravela \& Arregui et al. (2008).

Desde la década de los 90, se han desarrollado con fuerza las pruebas estandarizadas nacionales e internacionales en la región, algunos con discontinuidades y otros firmes en la causa como fin único y último de la educación; muestra de ello es el número de pruebas que se aplican en cada uno de los países de América Latina tanto de orden internacional como nacional (Tabla 2). 
Tabla 2. Países de América Latina y las pruebas estandarizadas.

\begin{tabular}{|c|c|}
\hline $\begin{array}{l}\text { Número de } \\
\text { países }\end{array}$ & Breve descripción \\
\hline Dieciséis & $\begin{array}{l}\text { Están participando en el Segundo Estudio Regional en } 3^{\circ} \text { y } 6^{\circ} \text { de primaria } \\
\text { que lleva adelante la OREALC/UNESCO: Argentina, Brasil, Chile, Colombia, } \\
\text { Costa Rica, Cuba, Ecuador, El Salvador, Guatemala, México, Nicaragua, Pa- } \\
\text { namá, Paraguay, Perú, República Dominicana y Uruguay. }\end{array}$ \\
\hline Seis & Participan en PISA 2006 y otros tres se incorporarían al ciclo PISA 2009. \\
\hline Algunos & $\begin{array}{l}\text { Países de la región han participado (o lo están haciendo), además, en los } \\
\text { estudios de Matemática y Ciencias (TIMSS), Lectura (PIRLS) y Educación } \\
\text { Cívica que lleva adelante la International Association for the Evaluation of } \\
\text { Educational Achievement (IEA). }\end{array}$ \\
\hline $\begin{array}{l}\text { Red de los sistemas } \\
\text { de evaluación }\end{array}$ & $\begin{array}{l}\text { Durante los últimos } 12 \text { años ha estado activa en la región la red de los siste- } \\
\text { mas de evaluación organizada en torno al Laboratorio Latinoamericano de } \\
\text { Evaluación de la Calidad de la Educación (LLECE) de OREALC/UNESCO, en } \\
\text { la que participan actualmente casi todos los países latinoamericanos. Esta } \\
\text { red se reúne dos veces al año y es un espacio de intercambio de experien- } \\
\text { cias y formación en el ámbito de la evaluación. }\end{array}$ \\
\hline $\begin{array}{l}\text { Grupo Iberoamericano } \\
\text { de PISA }\end{array}$ & $\begin{array}{l}\text { Integrado por Argentina, Brasil, Chile, Colombia, España, México, Portugal } \\
\text { y Uruguay. }\end{array}$ \\
\hline
\end{tabular}

Fuente: elaboración de los autores con información recabada de Ravela

$$
\text { \&Arregui et al. (2008). }
$$

Las evaluaciones estandarizadas internacionales en la región han aumentado y, desde luego, se espera que también los cambios de mejora se hayan observado en aras de la transparencia de los resultados y su difusión. Se han hecho reformas a los sistemas educativos y a los sistemas de evaluación, y las pruebas estandarizadas nacionales han aumentado y permeado todos los niveles de educación para estar en coherencia con las pruebas externas.

En la investigación de Gómez (2004), se puede inferir de manera directa en relación a que:

No se puede seguir haciendo comparaciones de calidad según el desempeño en las pruebas estandarizadas porque el resultado será siempre el mismo: las escuelas o instituciones educativas que no tienen la suerte de contar con estudiantes pertenecientes al quintil de más altos ingresos de la población y con mejor calidad de vida, se ubicarán siempre por debajo del nivel de desempeño mínimo esperado para dichas pruebas. Se debe tener presente, a la hora de hablar de calidad, 
que los insumos educativos - el nivel educativo de los padres, el entorno social donde se vive, los antecedentes culturales y socioeconómicos de los compañeros con que se estudia, la nutrición, los textos escolares, la preparación de los maestros y el acceso a nuevas tecnologías de la información y las comunicaciones, etc.-; determinan el rendimiento escolar. Entonces, es pertinente preguntarse: ¿se tienen en cuenta estos factores a la hora de diseñar las pruebas estandarizadas y de publicar los resultados de «los colegios de mayor desempeño»? (p. 87)

Las pruebas estandarizadas internacionales demarcan interés de orden político y económico e incluso de intervención de los organismos multilaterales, a pesar de que no evalúan del todo la realidad pluridiversa de cada contexto educativo y solo se encargan de medir a todos con la misma vara desconociendo las particularidades para clasificar, categorizar y de paso incluso excluir. Por lo tanto, no son pruebas incluyentes sino excluyentes, pues desconocen las situaciones propias -ambiente del sistema educativo de cada país y del mismo, sus diversas regiones y escenarios rurales y urbanos-.

En la perspectiva de Guevara (2017), se señala que:

Aunque se plantean cambios en la política educativa, desde los noventa se continúa con un modelo que confunde la evaluación con la medición y sigue dando importancia a los resultados del rendimiento de los estudiantes y a las pruebas estandarizadas. Existe una predominancia del discurso neoliberal, liderado por los organismos internacionales, con unos intereses claramente económicos y políticos. El Estado se encarga de dar razón de los avances de la política educativa y marca un lenguaje técnico empresarial "adecuado" a las instituciones educativas, que son las directas implicadas en rendir cuentas para dar o no continuidad a sus programas y proyectos institucionales (p. 168).

Y sin lugar a duda, se está en la misma línea de argumentación en tanto que la calidad de la educación dada por los resultados de las pruebas estandarizadas de orden homogéneo para una población heterogénea seguirá confundiendo la evaluación, la calidad con la medición cuantitativa lejos de la misma realidad social, económica, política y demás dimensiones de una sociedad que anhela su superación personal lejos de ser medidos por la misma vara.

\section{Pruebas estandarizadas a nivel internacional}

Entre las pruebas estandarizadas a nivel internacional más representativas en el sector de la educación se encuentran las pruebas PISA, SERCE y TIMMS; de manera que los resultados se usan para diseñar y/o implementar políticas educativas. 
Tabla 3. Pruebas estandarizadas internacionales.

\begin{tabular}{|c|c|}
\hline Prueba & Breve descripción \\
\hline PISA & $\begin{array}{l}\text { Es la sigla en inglés del Programa para la Evaluación Internacional de Alumnos. Se tra- } \\
\text { ta de un proyecto de la Organización para la Cooperación y el Desarrollo Económicos } \\
\text { (OCDE) es una Prueba internacional para evaluar la formación de los alumnos cuando } \\
\text { llegan al final de la etapa de enseñanza obligatoria, hacia los } 15 \text { años, los estudiantes } \\
\text { son seleccionados a partir de una muestra aleatoria. Busca ofrecer información detalla- } \\
\text { da que permita a los países participantes adoptar las decisiones necesarias para mejorar } \\
\text { los niveles educativos. Se realiza cada tres años. }\end{array}$ \\
\hline SERCE & $\begin{array}{l}\text { Segundo Estudio Regional Comparativo y Explicativo. El SERCE evaluó el logro de apren- } \\
\text { dizaje de 100,752 estudiantes de tercer grado y 95,288 de sexto grado de dieciséis } \\
\text { países más el Estado mexicano de Nuevo León, en Matemática, Lectura y Escritura y } \\
\text { Ciencias de la Naturaleza constituyendo, entonces, el estudio de calidad de la educación } \\
\text { más grande implementado en América Latina y el Caribe hasta esa fecha. El estudio, } \\
\text { además, indagó sobre los factores escolares y sociales que se asocian y posiblemente } \\
\text { explican el logro de los estudiantes. }\end{array}$ \\
\hline TIMMS & $\begin{array}{l}\text { El Estudio Internacional de Tendencias en Matemáticas y Ciencias (TIMSS, por sus siglas } \\
\text { en inglés) tiene como propósito medir las tendencias en el rendimiento de los estudiantes } \\
\text { de cuarto y octavo grado en Matemáticas y Ciencias. Ambas áreas son fundamentales } \\
\text { para desarrollar en los niños y jóvenes competencias relacionadas con la solución de } \\
\text { problemas y el razonamiento riguroso y crítico. Además, TIMSS monitorea la implemen- } \\
\text { tación de los currículos en estas áreas e identifica buenas prácticas de enseñanza para } \\
\text { aportar al mejoramiento de los procesos de enseñanza y aprendizaje. }\end{array}$ \\
\hline
\end{tabular}

Fuente: elaboración de los autores con información recabada de Ravela

\& Arregui et al. (2008).

Las pruebas estandarizadas PISA, SERCE, TIMMS, ICCS y PIRLS se han convertido en las pruebas más representativas y que miden el desempeño de los estudiantes a nivel internacional, por ello Colombia ha entrado en esta dinámica desde la década de los noventa como se evidencia en la Figura 1. Las de mayor aplicación son las pruebas PISA en cuatro ocasiones 2006, 2009, 2012 y 2015.

Figura 1. Pruebas Internacionales aplicadas en Colombia

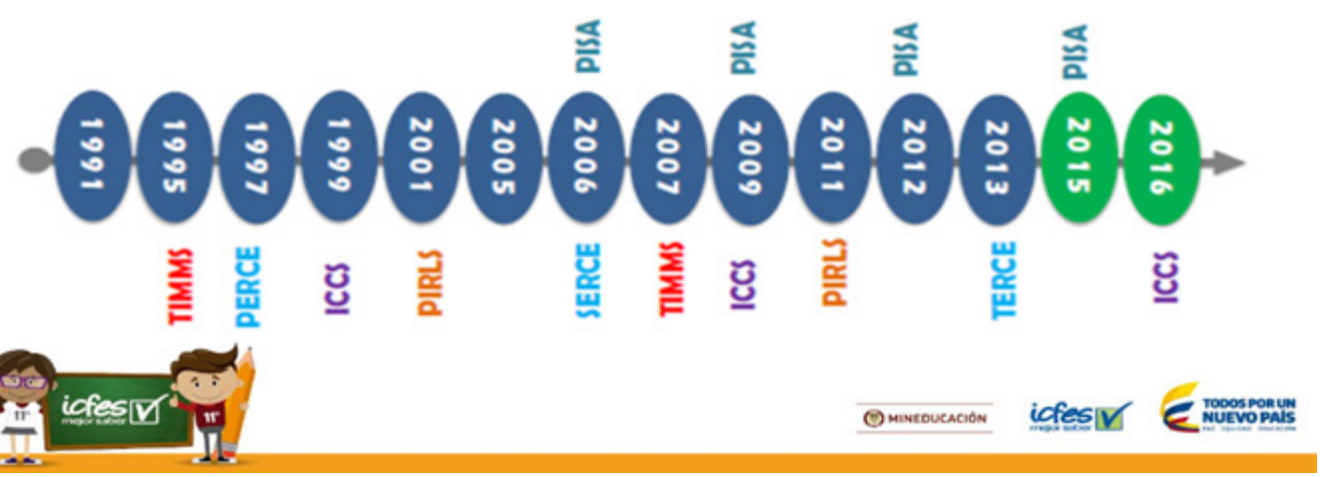

Fuente: Información recabada del Ministerio de Educación Nacional. 
Las pruebas estandarizadas internacionales que se aplican en Colombia, en coordinación con el Ministerio de Educación Nacional y el Instituto Colombiano para la Evaluación de la Educación (ICFES), en su pretensión de guardar armonía internacional en la educación y encuadrar en las políticas de los organismos multilaterales normalizan políticas y lineamientos de dichos organismos, incluso perdiendo o dejando de lado los intereses propios de cada contexto de Colombia y midiendo a todos por igual.

\section{Las pruebas estandarizadas en Colombia}

Como uno de los países de América Latina, Colombia no es ajena a las pruebas estandarizadas que se aplican en el mundo y también ha diseñado a través del ICFES un grupo de pruebas a lo largo de los niveles del sistema educativo.

Para Ayala-García, (2015), la evaluación en Colombia:

Recobra una línea diferente desde su creación en 1968, el ICFES ha sido la institución encargada de la evaluación de la educación en Colombia. Actualmente es un ente nacional independiente adscrito al Ministerio de Educación Nacional (MEN). Inicialmente, el ICFES realizaba solo lo que se denominaba «examen de Estado» para acceder a la educación superior. Luego se incluyeron otras pruebas como los exámenes de validación del bachillerato y las pruebas muestrales SABER (p. 7).

Con la Ley 115 de 1994 se dispuso la creación de un Sistema Nacional de Evaluación de la Educación a cargo del Ministerio de Educación de la mano del Servicio Nacional de Pruebas ICFES y las entidades territoriales (MEN, 2010).
También se sentaron las bases para el diseño e implementación de un nuevo sistema de evaluación. Desde el año 2002, el MEN comenzó a cambiar el enfoque de las evaluaciones nacionales, al pasar de evaluar conocimientos a la evaluación de competencias (MEN, 2010).

La educación superior no cuenta con evaluaciones nacionales formativas. La única prueba nacional que se aplica a los estudiantes universitarios es el examen Saber Pro, cuyo objetivo es evaluar la calidad de la educación superior en Colombia. Es de carácter censal y obligatorio para obtener el título de pregrado. También sirve de base para obtener información relevante para la toma de decisiones, rendición de cuentas por parte de las instituciones de educación superior y para comparaciones interinstitucionales.

Según el ICFES (2014),

Las evaluaciones que se realizan a través de pruebas estandarizadas deben basarse en instrumentos válidos y confiables para que sea posible hacer inferencias apropiadas sobre los conocimientos, habilidades y capacidades de las personas evaluadas. Una prueba es válida cuando mide lo que se quiere medir, es decir, que hay consistencia entre lo que se pregunta en la prueba y el referente que se definió para la evaluación. A su vez, una prueba es confiable cuando permite hacer mediciones precisas sobre lo que se está evaluando; la validez y la confiabilidad son elementos esenciales para producir resultados que describan, con un alto nivel de precisión, las competencias de las personas evaluadas.

Se puede evidenciar que las pruebas diseñadas y aplicadas por el ICFES se estructuran bajo la metodología denominada diseño de especificaciones, a partir del diseño centrado en 
evidencias. En síntesis, se trata de un conjunto de prácticas de desarrollo de instrumentos que busca definir lo que mide una prueba y apoyar las inferencias que se hacen con base en las evidencias derivadas de esta. Con ello, se busca asegurar la validez mediante la alineación de los procesos y productos de las pruebas con sus objetivos y ahí se pueden ubicar a las pruebas Saber Pro.

En Colombia también se evidencia la organización de las pruebas estandarizadas y se denominan Saber, como especifica el periódico Altablero (2006):

Se aplica a estudiantes de $5^{\circ}$ y $9^{\circ}$ grado. Comenzó en 1991 con aplicaciones muestrales y entre 2002 y 2003 se llevó a cabo la primera aplicación censal, que constituye una línea de base en las áreas de Lenguaje, Matemáticas, Ciencias Naturales y Competencias Ciudadanas. A partir de 2005 se incluyó Ciencias Sociales.

Con el paso del tiempo se ha ampliado la cobertura y se aplican a los estudiantes en los diferentes niveles de la educación. Saber $3^{\circ}$ y $5^{\circ}$ corresponde a la Básica Primaria, $9^{\circ}$ a Básica Secundaria, Saber $11^{\circ}$ a la Media Vocacional y Saber Pro la educación superior en la formación profesional (Tabla 4).

\section{Tabla 4. Pruebas estandarizadas nacionales.}

\begin{tabular}{|c|c}
\multicolumn{1}{c|}{ Prueba } & \multicolumn{1}{c}{ Breve descripción } \\
\hline SABER $\mathbf{3}^{\circ}, \mathbf{5}^{\circ}$ y $\mathbf{9}^{\circ}$ & $\begin{array}{l}\text { Tienen aplicaciones periódicas en los grados tercero, quinto y noveno, para medir la } \\
\text { calidad de los niveles de básica primaria y secundaria, respectivamente. }\end{array}$ \\
\hline SABER $11^{\circ}$ & $\begin{array}{l}\text { La prueba SABER } 11^{\circ} \text {, evaluación del nivel de la Educación Media a partir del año } \\
\text { 2014, se alinea con las evaluaciones de la Educación Básica para proporcionar in- } \\
\text { formación a la comunidad educativa en el desarrollo de las competencias básicas } \\
\text { que debe desarrollar un estudiante durante el paso por la vida escolar; además de } \\
\text { ser una herramienta que retroalimenta al Sistema Educativo }\end{array}$ \\
\hline SABER PRO & $\begin{array}{l}\text { Busca comprobar el grado de desarrollo de competencias en estudiantes próxi- } \\
\text { mos a culminar su educación superior. Produce indicadores de valor agregado de } \\
\text { la educación superior y compara las competencias existentes antes de ingresar y } \\
\text { al terminar la carrera. Por lo tanto, sirve como fuente de información para construir } \\
\text { indicadores de evaluación a la calidad de programas e instituciones. }\end{array}$ \\
\hline
\end{tabular}

Fuente: Elaboración de los autores con información recabada de Celis, Jiménez y Jaramillo, 2006. 
En Colombia existe un sistema de pruebas estandarizadas que realiza el ICFES a las que se denomina:

Pruebas Saber, las cuales tienen aplicaciones periódicas en los grados quinto y noveno (para medir la calidad de los niveles de básica primaria y secundaria, respectivamente), y en el grado undécimo, antes llamada examen de Estado para el ingreso a la educación superior, la más antigua y utilizada por Instituciones de Educación Superior (IES) del país como un criterio de selección de sus admitidos. Recientemente se aplica el examen Saber Pro (antes Ilamada Ecaes), a estudiantes que están culminando el ciclo técnico, tecnológico o profesional y cuya finalidad es evaluar la calidad de la educación superior en Colombia (Celis, Jiménez y Jaramillo, 2006).

El objeto de estudio de la investigación se centra en las pruebas Saber Pro de la formación de profesionales en educación, en el marco de una licenciatura que se oferta en la modalidad a distancia y cuyo periodo de análisis de evidencia para el año 2013.

\section{Materiales y métodos}

El proyecto de investigación se enmarca en el enfoque mixto no experimental de tipo descriptivo, puesto que da cuenta de los resultados de las pruebas Saber Pro de los estudiantes en el año 2013. En la Tabla 5 se describe la muestra seleccionada, la cual contó con la participación de estudiantes matriculados de noveno y décimo semestre de la licenciatura. Se trata de una población heterogénea compuesta por jóvenes entre 18 y 25 años provenientes de diferentes regiones del país, de la cual un gran porcentaje son mujeres.

\begin{tabular}{l|c|c} 
Tabla 5. Muestra en función del género \\
de los estudiantes participantes. \\
Género & Muestra & Porcentaje \\
\hline Femenino & 198 & $73.3 \%$ \\
\hline Masculino & 72 & $26.6 \%$ \\
\hline Total & 270 & $100 \%$ \\
\hline
\end{tabular}

Fuente: archivo de la investigación.

La población objeto de estudio fue de 270 estudiantes de los Centros Regionales de Educación a Distancia (Tunja, Duitama, Sogamoso, Chiquinquirá y Bogotá) que presentaron dichas pruebas. Una vez caracterizado el grupo, se evidencia que la mayoría de estudiantes son de sexo femenino con un $73.3 \%$ del total por encima del grupo de sexo masculino con un $26.6 \%$, lo que indica que la mayoría de estudiantes en el grupo de estudio son de sexo femenino.

\section{Resultados y discusión}

En adelante, se presenta un análisis estadístico descriptivo de las pruebas que presentaron los estudiantes del programa de Licenciatura en Educación Básica con Énfasis en Matemáticas, Humanidades y Lengua Castellana de la Facultad de Estudios a Distancia de la Universidad Pedagógica y Tecnológica de Colombia en el año 2013. El análisis versa en dos sentidos en la misma dirección. Por una parte, se presentan y analizan los resultados de las competencias genéricas evaluadas en el año 2013 y, por otra parte, se muestran y estudian las derivaciones de los resultados de los módulos específicos en Educación para el mismo año. 


\section{Resultados y análisis de competencias genéricas año 2013}

Los estudiantes de la Licenciatura en Educación Básica con énfasis en Matemáticas, Humanidades y Lengua Catellana que presentaron las pruebas Saber Pro en el año 2013, fueron objeto de evaluación homogénea y estándar en las siguientes competencias y componentes: Competencias Ciudadanas. Comunicación Escrita, Inglés, Lectura Crítica, Razonamiento Cuantitativo, cuyos resultados y análisis se evidencian en las tablas y gráficas subsiguientes.

Tabla 6. Quintil del Módulo de Escritura Año 2013.

\begin{tabular}{c|c|c|c|c} 
Quintil & Frecuencia & Porcentaje & Porcentaje válido & Porcentaje acumulado \\
\hline I & 36 & 13 & 13 & 13 \\
\hline II & 62 & 23 & 23 & 36 \\
\hline III & 75 & 28 & 28 & 64 \\
\hline IV & 50 & 18 & 18 & 82 \\
\hline V & 49 & 18 & 18 & $100 \%$ \\
\hline Total & $\mathbf{2 7 2}$ & $\mathbf{1 0 0 \%}$ & &
\end{tabular}

Fuente: archivo de la investigación.

La Tabla 6 presenta la clasificación de estudiantes de acuerdo con el quintil dentro del grupo de referencia educación. Se observa que el 64\% de los estudiantes del programa Licenciatura en Educación Básica con énfasis en Matemáticas, Humanidades y Lengua Castellana hace parte del $60 \%$ de los estudiantes con menores puntajes dentro del grupo de referencia. El $36 \%$ restante hace parte del 40\% con mayores puntajes.

Figura 2. Quintil Módulo Escritura.

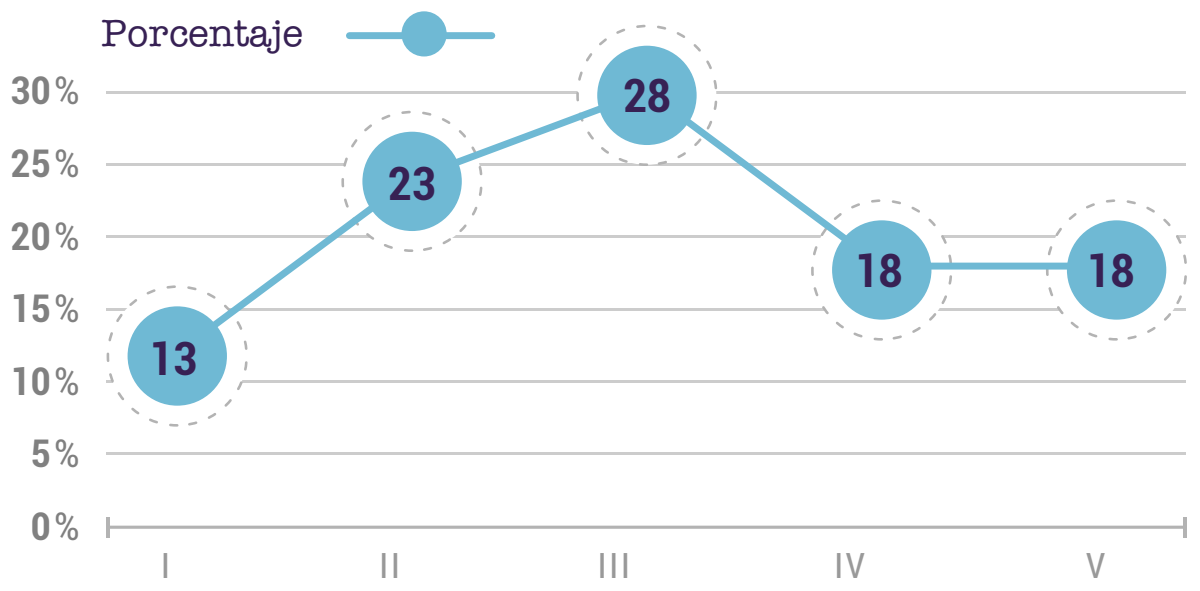

Fuente: Archivo de la investigación 
En la Figura 2 se presenta el comportamiento de los porcentajes de estudiantes en el Módulo de Escritura. Se muestra una mayor concentración de porcentaje de estudiantes en los quintiles I, II y III. Decrecen en el quintil IV y permanecen constantes hasta el quintil $\mathrm{V}$.

Tabla \%. Nivel de desempeño del Módulo Escritura Año 2013.

\begin{tabular}{c|c|c|c|c} 
Niveles & Frecuencia & Porcentaje & Porcentaje válido & Porcentaje acumulado \\
\hline 1 & 2 & 1 & 1 & 1 \\
\hline 2 & 7 & 3 & 3 & 4 \\
\hline 3 & 28 & 10 & 10 & 14 \\
\hline 4 & 111 & 41 & 41 & 55 \\
\hline 5 & 82 & 30 & 30 & 85 \\
\hline 6 & 32 & 11 & 11 & 96 \\
\hline 7 & 10 & 4 & 4 & $100 \%$ \\
\hline Total & $\mathbf{2 7 2}$ & $\mathbf{1 0 0} \%$ & & \\
\hline
\end{tabular}

Fuente: Archivo de la investigación.

Los desempeños del Módulo de Escritura se clasifican en 8 niveles acumulativos y jerárquicos. En la Tabla 7 se muestra el porcentaje de estudiantes del programa de Licenciatura en Educación Básica con Énfasis en Matemáticas, Humanidades y Lengua Castellana pertenecientes a cada nivel. En el nivel 1 se encuentran el 1\% de los estudiantes del programa, que presentan dificultad en el manejo de expresiones básicas de concordancia en la escritura. En el nivel 2 se encuentra el $4 \%$ de los estudiantes, que presentan problemas en la coherencia y articulación de escritos. En el nivel 3 está el 10\% de los estudiantes, que de una u otra manera tienen inconveniente con el dominio de las habilidades comunicativas. En el nivel 4 está el $41 \%$ de los estudiantes que, si bien presentan avances en el lenguaje, aún persisten en una mala organización de las ideas en un texto escrito. En el nivel 5 está el $30 \%$ de los dicentes que alcanzan una coherencia en el texto y pueden establecer relaciones entre las ideas. En el nivel 6 se encuentra el $11 \%$ de los estudiantes que hacen uso adecuado y articulado del lenguaje. En el nivel 7 está solo el $4 \%$, de los estudiantes 
es capaz de establecer un dominio del lenguaje y adecuarlo para un determinado público y en el nivel 8 no hay estudiantes clasificados.

Figura 3. Nivel de desempeño Módulo Escritura.

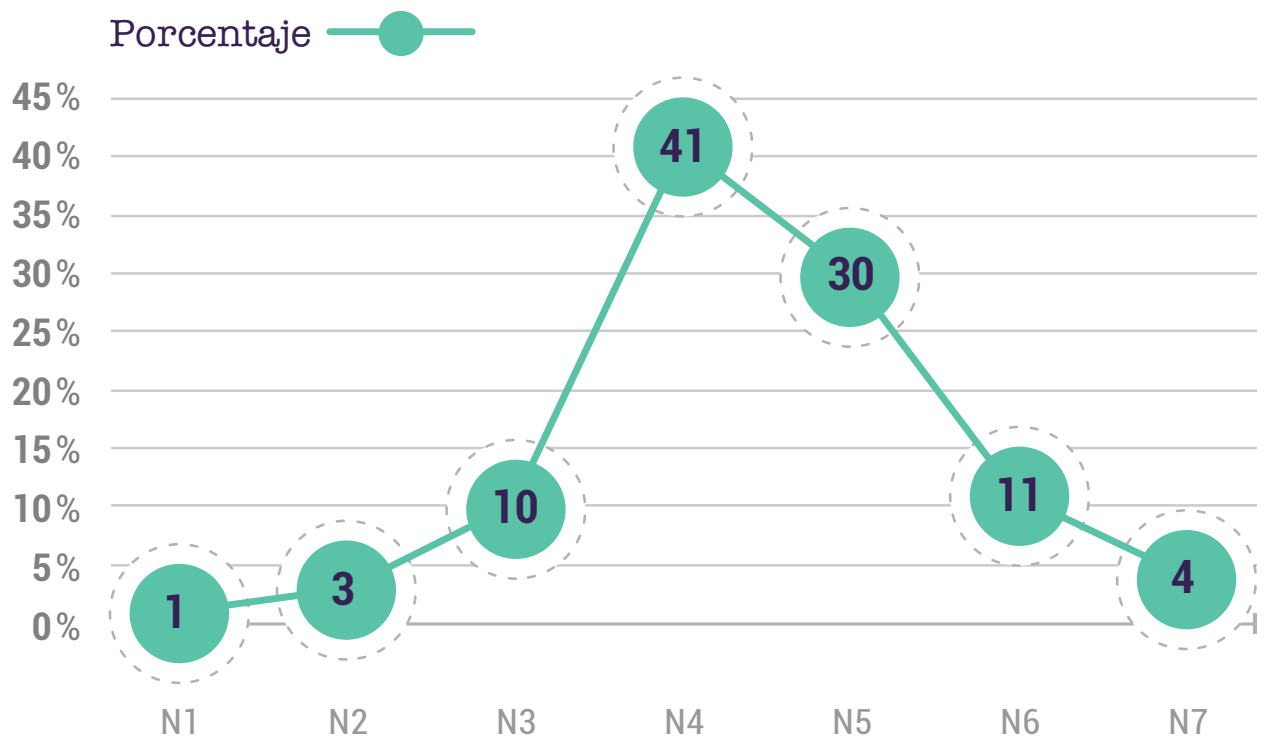

Fuente: Archivo de la investigación.

En la Figura 3 se presenta el comportamiento de los porcentajes de estudiantes en el Módulo de Escritura. Hay un comportamiento creciente en los cuatro primeros niveles, luego empieza a descender hasta llegar al nivel 7 . El ideal es que más porcentajes de estudiantes se concentren en los niveles 5, 6 y 7.

Tabla 8. Quintil Módulo Razonamiento Cuantitativo Año 2013.

\begin{tabular}{c|c|c|c|c} 
Quintil & Frecuencia & Porcentaje & Porcentaje válido & Porcentaje acumulado \\
\hline I & 56 & 21 & 21 & 21 \\
\hline II & 61 & 22 & 22 & 43 \\
\hline III & 65 & 24 & 24 & 67 \\
\hline IV & 48 & 18 & 18 & 85 \\
\hline V & 42 & 15 & 15 & $100 \%$ \\
\hline Total & $\mathbf{2 7 2}$ & $\mathbf{1 0 0} \%$ & & \\
\hline
\end{tabular}

Fuente: Archivo de la investigación.

La tabla 8 muestra que el $67 \%$ de los estudiantes del programa de Licenciatura en Educación Básica con Énfasis en Matemáticas, Humanidades y Lengua Castellana hacen parte del $60 \%$ de los estudiantes 
con los menores puntajes dentro del grupo de referencia Educación. Es decir, presentan dificultad para comprender y manipular información presentada en diversos formatos, plantear estrategias adecuadas para resolver un problema, utilizar estrategias matemáticas para dar solución a un problema.

El 33\% restante de los estudiantes del programa de Licenciatura en Educación Básica hacen parte del $40 \%$ con mayores puntajes dentro del grupo de referencia Educación. Es de resaltar que el 15\% de los estudiantes están clasificados en el quintil V.

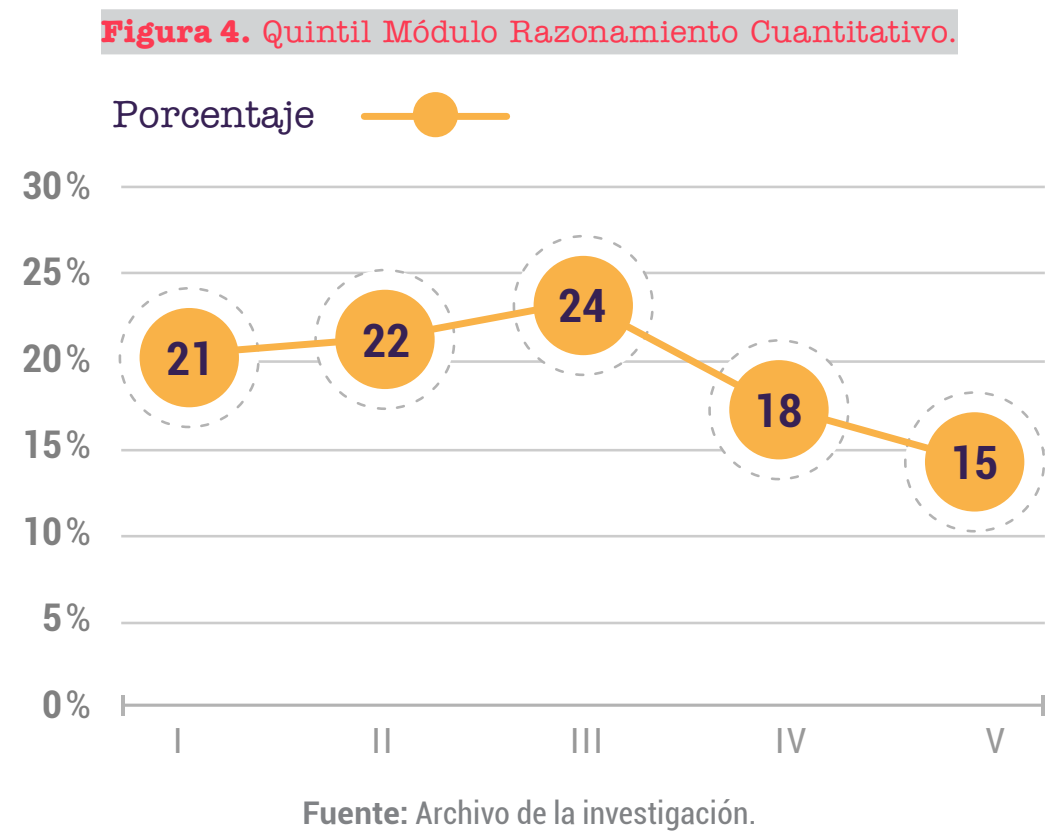

En la Figura 4 se observa que hay un incremento de los quintiles I, II y III de porcentajes de estudiantes concentrados allí. Se evidencia también un decremento en los quintiles IV y V.

Tabla 9. Quintil Módulo Lectura Crítica Año 2013.

\begin{tabular}{c|c|c|c|c} 
Quintil & Frecuencia & Porcentaje & Porcentaje válido & Porcentaje acumulado \\
\hline I & 63 & 23 & 23 & 23 \\
\hline II & 66 & 24 & 24 & 47 \\
\hline III & 72 & 27 & 27 & 74 \\
\hline IV & 39 & 14 & 14 & 88 \\
\hline V & 32 & 12 & 12 & $100 \%$ \\
\hline Total & $\mathbf{2 7 2}$ & $\mathbf{1 0 0} \%$ & &
\end{tabular}

Fuente: Archivo de la investigación. 
En la Tabla 9 se muestra que el $74 \%$ de los estudiantes del programa de Licenciatura en Educación Básica con Énfasis en Matemáticas, Humanidades y Lengua Castellana hacen parte del $60 \%$ de los estudiantes con puntajes bajos dentro del grupo de referencia Educación. Se puede inferir que dicho grupo de estudiantes evidencian algún inconveniente en el manejo de las estructuras literarias y escriturales propias de la lengua castellana. El $26 \%$ restante de los estudiantes del programa de Licenciatura en Educación Básica hacen parte del $40 \%$ de los estudiantes con mayores puntajes dentro del grupo de referencia Educación.

\section{Figura 5. Quintil Módulo Lectura Crítica.}
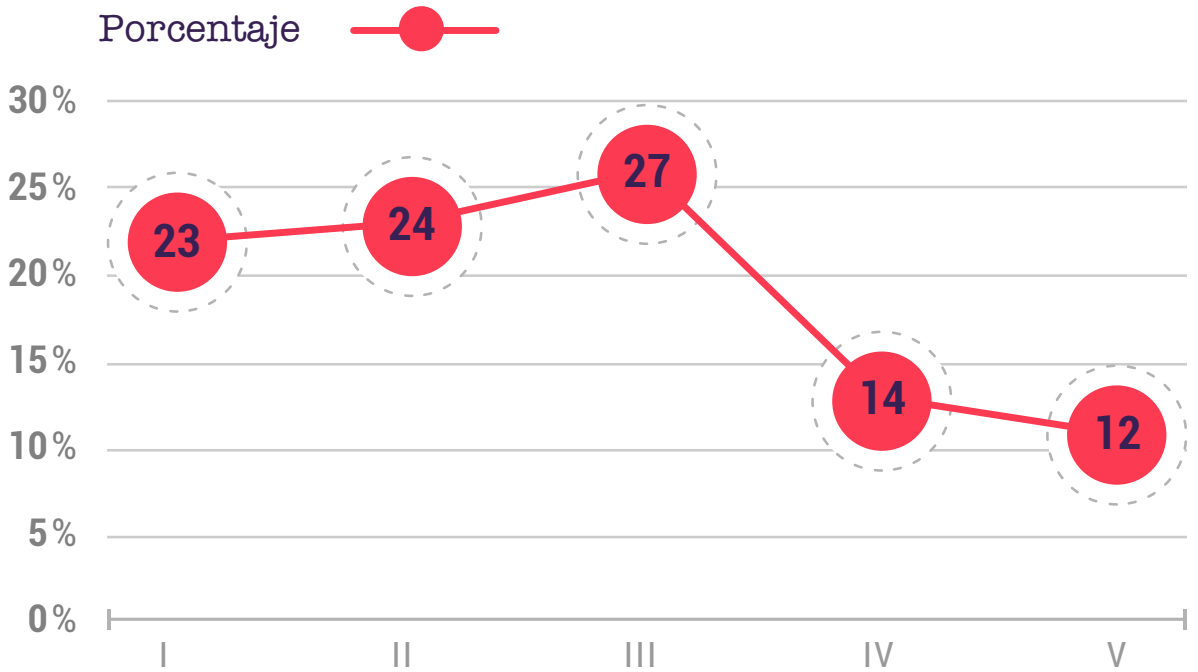

Fuente: Archivo de la investigación.

En la Figura 5 se observa una gran concentración de porcentaje de estudiantes en los quintiles I, II y III, mostrándose un decrecimiento en los quintiles IV y V. Para que los resultados fueran óptimos se debería tener un menor número de porcentajes de estudiantes ubicados en dichos quintiles.

Tabla 10. Quintil Módulo Competencias Ciudadanas Año 2013.

\begin{tabular}{c|c|c|c|c} 
Quintil & Frecuencia & Porcentaje & Porcentaje válido & Porcentaje acumulado \\
\hline I & 46 & 17 & 17 & 17 \\
\hline II & 63 & 23 & 23 & 40 \\
\hline III & 70 & 26 & 26 & 66 \\
\hline IV & 46 & 17 & 17 & 83 \\
\hline V & 47 & 17 & 17 & $100 \%$ \\
\hline Total & $\mathbf{2 7 2}$ & $\mathbf{1 0 0} \%$ & &
\end{tabular}

Fuente: Archivo de la investigación. 
En la Tabla 10 se observa que el $66 \%$ de los estudiantes del programa Licenciatura en Educación Básica con énfasis en Matemáticas, Humanidades y Lengua Castellana obtuvieron puntajes por debajo de la media del grupo de referencia, lo que indica que existe un desconocimiento de los deberes y derechos como ciudadanos. De igual manera el $34 \%$ restante forma parte del $40 \%$ con mayores puntajes

Figura 6. Quintil Módulo Competencias Ciudadanas.
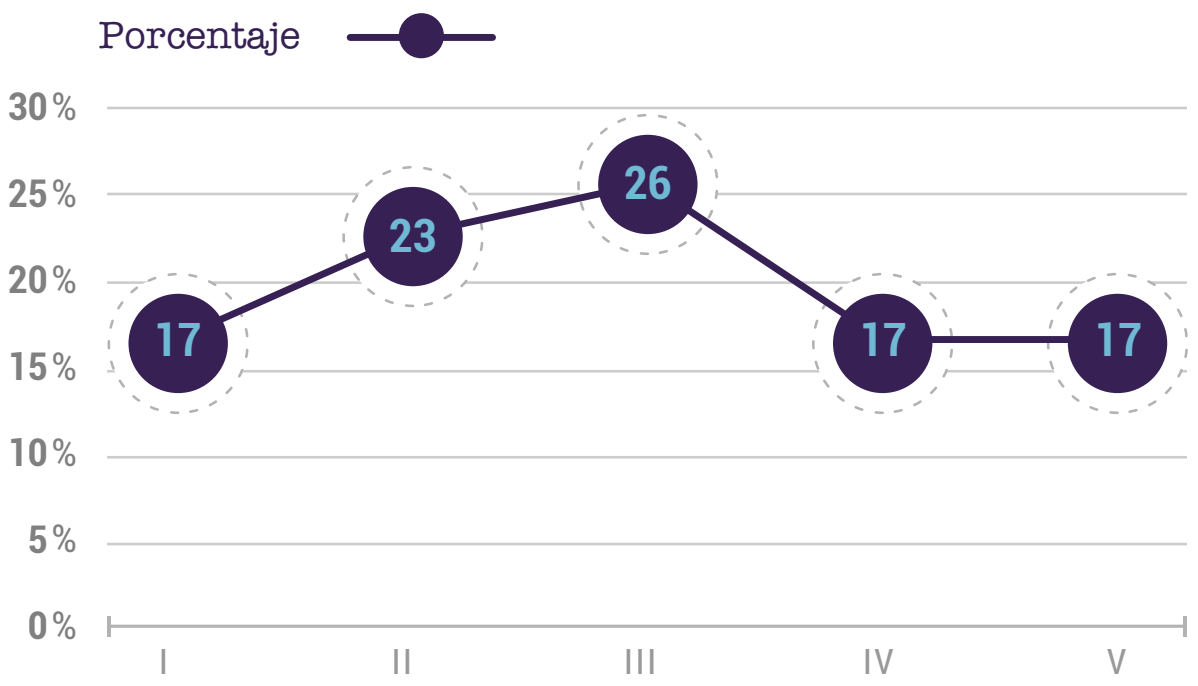

Fuente: Archivo de la investigación.

En la Figura 6 se observa el comportamiento de los porcentajes en el Módulo de Competencias Ciudadanas, se evidencia que los puntajes más bajos corresponden a los quintiles I, II y III siendo el quintil III el de mayor porcentaje. Asimismo, se presenta una disminución en el quintil $\mathrm{IV}$, permaneciendo constante hasta el quintil V.

Tabla 11. Quintil Módulo Inglés Año 2013.

\begin{tabular}{c|c|c|c|c} 
Quintil & Frecuencia & Porcentaje & Porcentaje válido & Porcentaje acumulado \\
\hline I & 57 & 21 & 21 & 21 \\
\hline II & 77 & 28 & 28 & 49 \\
\hline III & 64 & 23 & 23 & 72 \\
\hline IV & 59 & 22 & 22 & 84 \\
\hline V & 15 & 6 & 6 & $100 \%$ \\
\hline Total & $\mathbf{2 7 2}$ & $\mathbf{1 0 0 \%}$ & &
\end{tabular}

Fuente: Archivo de la investigación. 
En la Tabla 11 se aprecia la clasificación en quintiles para el Módulo de Inglés. El $72 \%$ de los estudiantes del programa de Licenciatura en Educación Básica con Énfasis en Matemáticas, Humanidades y Lengua Castellana hacen parte del $60 \%$ de los estudiantes con los menores puntajes dentro del grupo de referencia Educación. El 28\% hace parte del $40 \%$ con mayores puntajes. Es de resaltar que existen estudiantes clasificados en el quintil $V$.

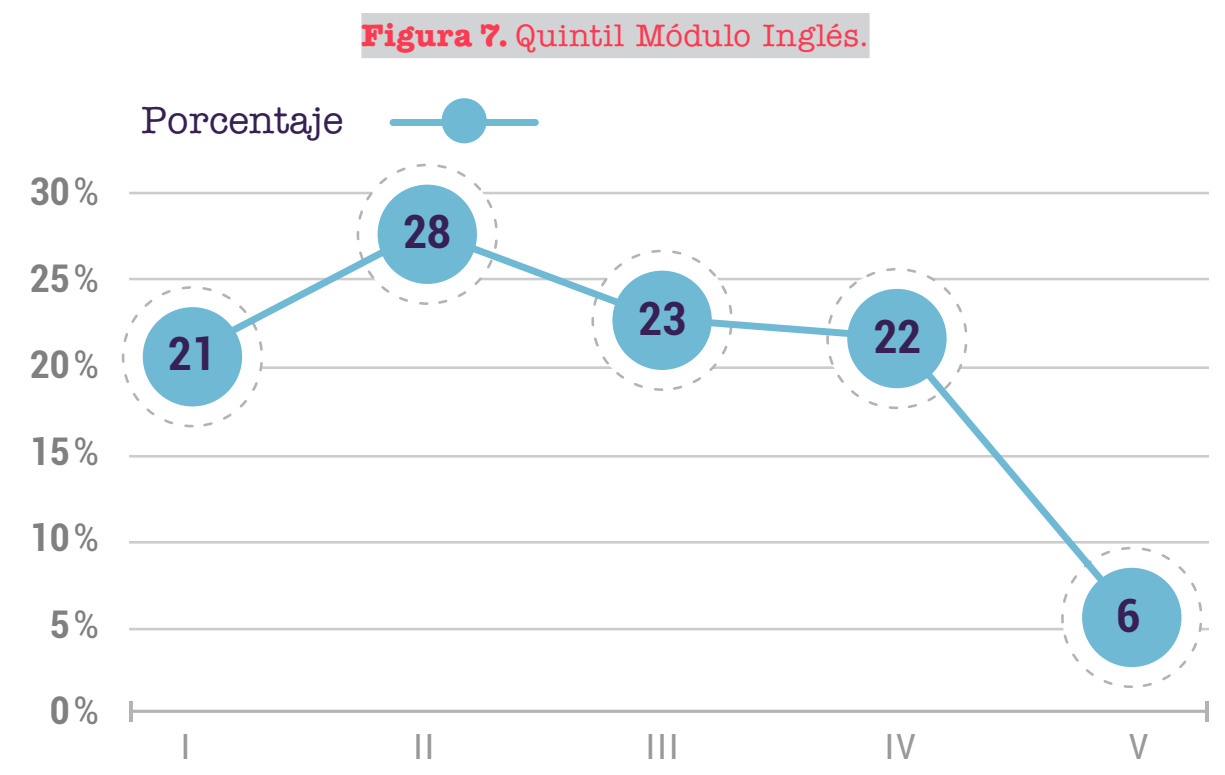

Fuente: Archivo de la investigación.

La Figura 7 muestra un alto número de porcentaje de estudiantes ubicados del quintil I al III y posteriormente se presenta una poca concentración de los mismos en los quintiles IV y V, lo que hace que muy pocos estudiantes alcancen los niveles más altos de desempeño.

Tabla 12. Nivel de Desempeño Inglés Año 2013.

\begin{tabular}{c|c|c|c|c} 
Nivel de desempeño & Frecuencia & Porcentaje & Porcentaje válido & Porcentaje acumulado \\
\hline A- & 97 & 36 & 36 & 36 \\
\hline A1 & 123 & 45 & 45 & 81 \\
\hline A2 & 42 & 15 & 15 & 96 \\
\hline B1 & 8 & 3 & 3 & 99 \\
\hline B+ & 2 & 1 & 1 & $100 \%$ \\
\hline B2 & 0 & 0 & 0 & $100 \%$ \\
\hline TOTAL & $\mathbf{2 7 2}$ & $\mathbf{1 0 0 \%}$ & & \\
\hline
\end{tabular}

Fuente: Archivo de la investigación. 
En la Tabla 12 se observa que el $36 \%$ de los estudiantes están en el nivel de desempeño $A$, esta clasificación indica que no alcanzan el nivel A1. El $45 \%$ está en el nivel A1, es decir, que los estudiantes son capaces de comprender y utilizar expresiones cotidianas de uso muy frecuente, así como frases sencillas destinadas a satisfacer necesidades de tipo inmediato; pueden presentarse ellos mismos y a otros, pedir y dar información básica sobre su domicilio, sus pertenencias y las personas que conoce.

El 15\% está en el nivel A2, los estudiantes saben comunicarse a la hora de llevar a cabo tareas simples y cotidianas que no requieran más que intercambios sencillos y directos de información sobre cuestiones que le son conocidas o habituales. El 3\% está en el nivel B1, en este nivel los estudiantes son capaces de producir textos sencillos y coherentes sobre temas que le son familiares o en los que tiene un interés personal y, finalmente, el $1 \%$, es decir, un estudiante alcanza el nivel B+. En el nivel B2 no hay estudiantes clasificados.

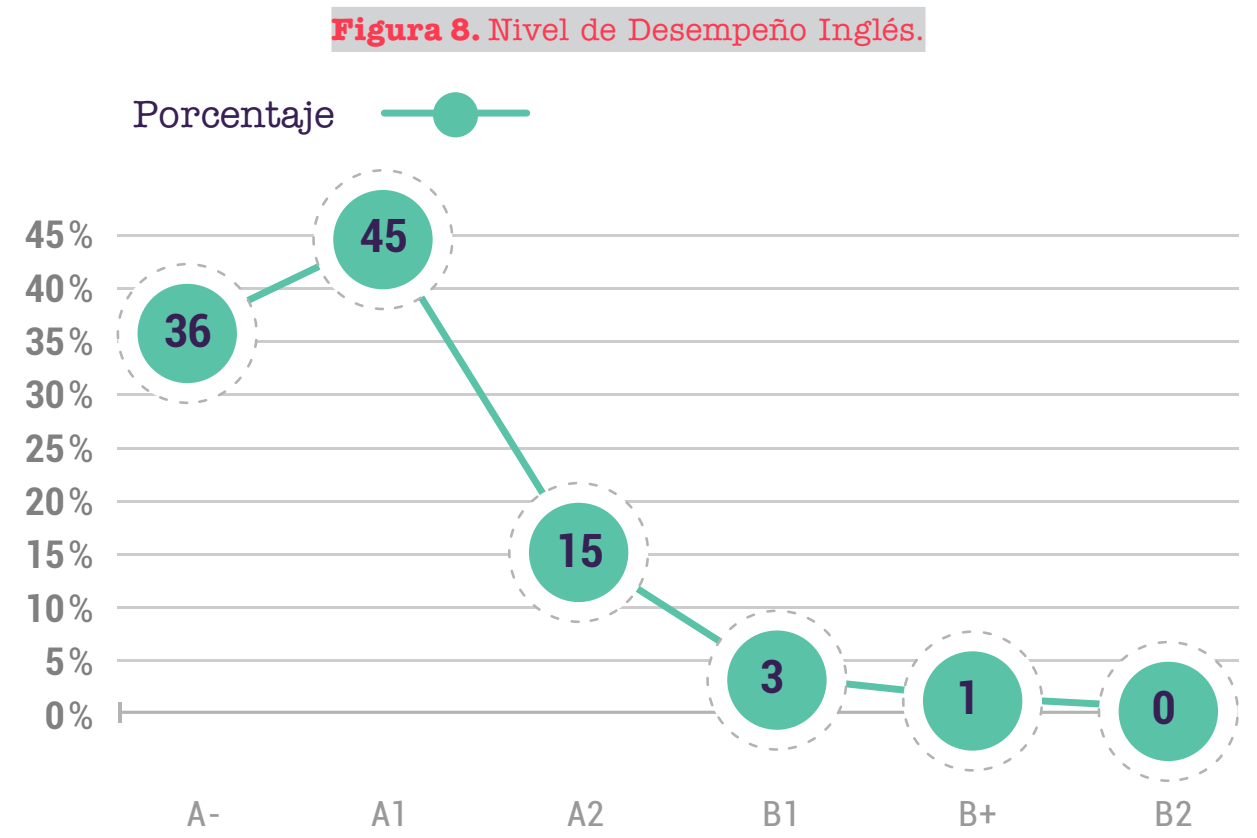

Fuente: Archivo de la investigación.

En la Figura 8 se observa una gran concentración de porcentajes de estudiantes en los niveles A-, A1 y A2. Luego se muestra un porcentaje muy bajo de estudiantes en los niveles de desempeño B1 y B+. Asimismo, se puede evidenciar que en el nivel máximo de desempeño no se encuentra ningún estudiante. 
Tabla 13. Comparativo Programa, Grupo de Referencia

y Nacional Pruebas Genéricas.

\begin{tabular}{l|c|c|c|c|c|c|c|c|c}
\multicolumn{1}{c}{ Modulo } & \multicolumn{2}{c}{ Programa } & \multicolumn{3}{c}{ Grupo de referencia } & \multicolumn{3}{c}{ Nacional } \\
\hline Com. Escrita & N & Promedio & N & Promedio & Desviación & N & Promedio & Desviación \\
\hline Raz. Cuantitativo & 270 & 10.1 & 22439 & 10.2 & 1.1 & 183217 & 9.9 & 1 \\
\hline Lec. Critica & 270 & 10.05 & 22439 & 10.4 & 0.9 & 183217 & 9.5 & 1.2 \\
\hline Comp. Ciudadanas & 270 & 9.7 & 22439 & 10.2 & 1 & 183217 & 9.8 & 1 \\
\hline Inglés & 270 & 10.4 & 22439 & 9.86 & 1 & 183217 & 9.5 & 1 \\
\hline & 270 & 10.15 & 22439 & 10.3 & 1.2 & 183217 & 9.8 & 1.1 \\
\hline
\end{tabular}

Fuente: Archivo de la investigación

En la Tabla 13 se observan los promedios nacionales, grupo de referencia y del programa de los cinco módulos de competencias genéricas. En la tabla los puntajes del programa varían de un mínimo de 9.7 en el Módulo de Lectura Crítica a un máximo de 10.4 en el Módulo de Competencias Ciudadanas. Asimismo, los del grupo de referencia varían de un mínimo de 9.86 en el módulo de Competencias Ciudadanas a un máximo de 10.4 en Razonamiento cuantitativo. De igual forma, el promedio nacional varía de un mínimo de 9.5 en el Módulo de Razonamiento Cuantitativo a un máximo de 9.9 en el Módulo de Comunicación Escrita.

Figura 9. Comparativo de Programa, Grupo de Referencia y Nacional.

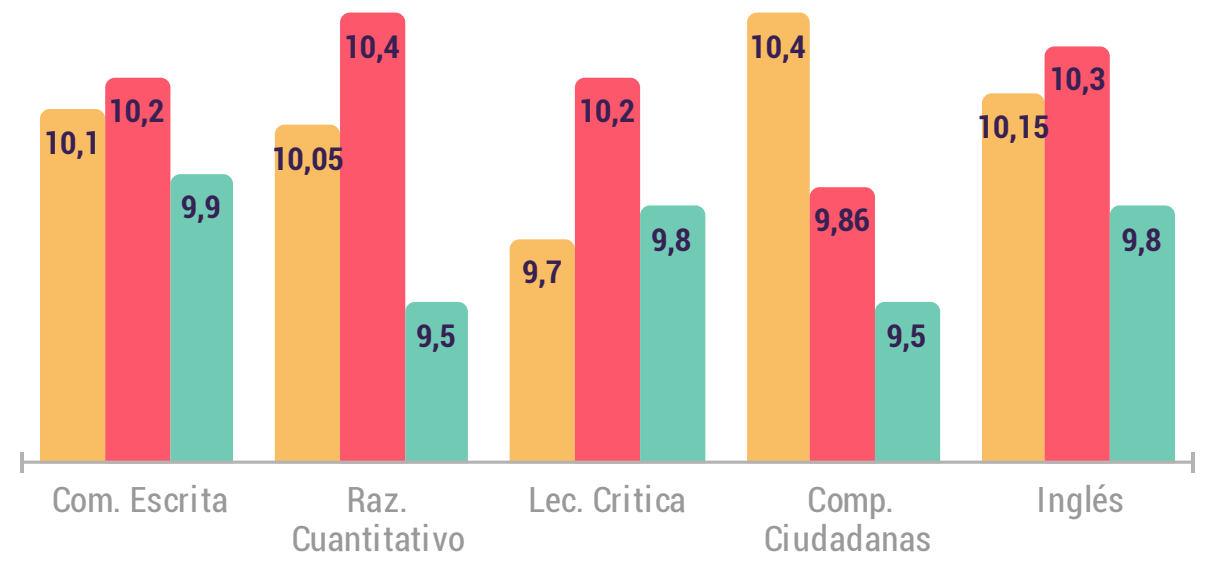

Programa $\quad$ Grupo de referencia $\quad$ Nacional

Fuente: Archivo de la investigación. 
En la Figura 9 se observa el desempeño de los estudiantes del programa frente a los promedios del grupo de referencia y nacional. En el Módulo de Comunicación Escrita el promedio del programa está por debajo del promedio del grupo de referencia, pero por encima del promedio nacional. Con relación al Módulo de Razonamiento Cuantitativo se observa que el promedio del programa está por debajo del promedio del grupo de referencia, pero por encima del nacional. En Lectura Crítica el promedio del programa se encuentra por debajo del promedio del grupo de referencia y por debajo del promedio nacional. En Competencias Ciudadanas el promedio del programa se encuentra por encima del promedio del grupo de referencia y nacional y, por último, con respecto al Módulo de Inglés, el promedio del programa está por debajo del promedio del grupo de referencia, pero por encima del promedio nacional.

\section{Resultados y análisis de los módulos específicos: Educación para el año 2013}

Los estudiantes de la Licenciatura en Educación Básica con enfasis en Matemáticas, Humanidades y Lengua Catellana adscrita a la Facultad de Estudios a Distancia de la Universidad Pedagógica y Tecnologica de Colombia, que presentaron las pruebas Saber Pro en el año 2013, fueron objeto de evaluación en condiciones estandarizadas para efectos de medición en los módulos específicos de Educación a saber: Enseñar, Evaluar y Formar.

Tabla 14. Quintil Módulo Enseñar Año 2013.

\begin{tabular}{c|c|c|c|c} 
Quintil & Frecuencia & Porcentaje & Porcentaje válido & Porcentaje acumulado \\
\hline I & 50 & 18 & 18 & 18 \\
\hline II & 67 & 25 & 25 & 43 \\
\hline III & 67 & 25 & 25 & 68 \\
\hline IV & 53 & 19 & 19 & 87 \\
\hline V & 35 & 13 & 13 & $100 \%$ \\
\hline Total & $\mathbf{2 7 2}$ & $\mathbf{1 0 0 \%}$ & & \\
\hline
\end{tabular}

Fuente: Archivo de la investigación. 
En la Tabla 14 se observa que el $68 \%$ de los estudiantes del programa de Licenciatura en Educación Básica con Énfasis en Matemáticas, Humanidades y Lengua Castellana hacen parte del $60 \%$ de los estudiantes con los menores puntajes y el $32 \%$ restante del $40 \%$ de los estudiantes con mejores puntajes.

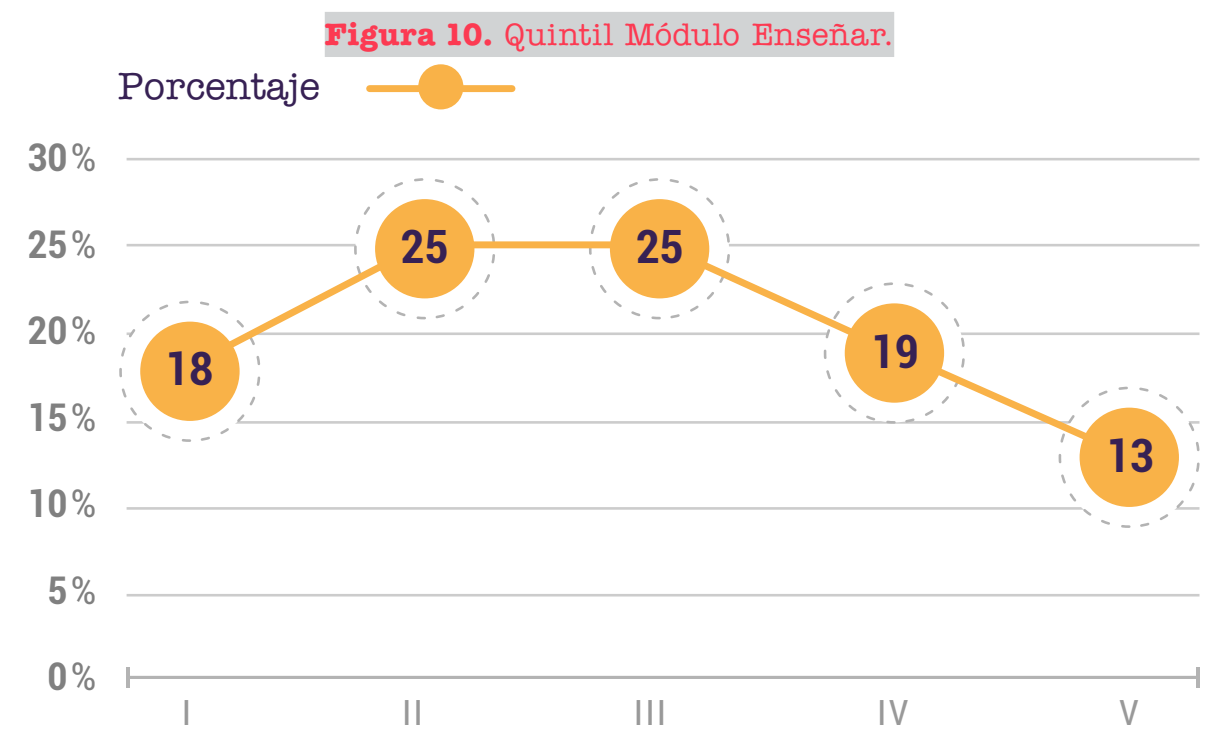

Fuente: Archivo de la investigación.

En la Figura 10 se observa un gran número de porcentaje de estudiantes en los quintiles I, II y III. Asimismo, en los quintiles IV y V existen muy pocos estudiantes que alcanzaron estos niveles de desempeño.

Tabla 15. Quintil Módulo Evaluar Año 2013.

\begin{tabular}{c|c|c|c|c} 
Quintil & Frecuencia & Porcentaje & Porcentaje válido & Porcentaje acumulado \\
\hline I & 51 & 19 & 19 & 19 \\
\hline II & 67 & 25 & 25 & 44 \\
\hline III & 66 & 24 & 24 & 68 \\
\hline IV & 54 & 19 & 19 & 87 \\
\hline V & 34 & 13 & 13 & $100 \%$ \\
\hline Total & $\mathbf{2 7 2}$ & $\mathbf{1 0 0} \%$ & &
\end{tabular}

Fuente: Archivo de la investigación.

En la Tabla 15 se observa la clasificación del porcentaje de estudiantes de acuerdo con el quintil dentro del grupo de referencia educación para el Módulo Evaluar. El 68\% de los estudiantes del programa de Licenciatura en Educación Básica con Énfasis en Matemáticas, 
Humanidades y Lengua Castellana hacen parte del $60 \%$ de los estudiantes con los menores puntajes y el $32 \%$ restante del $40 \%$ de los estudiantes con puntajes más altos.

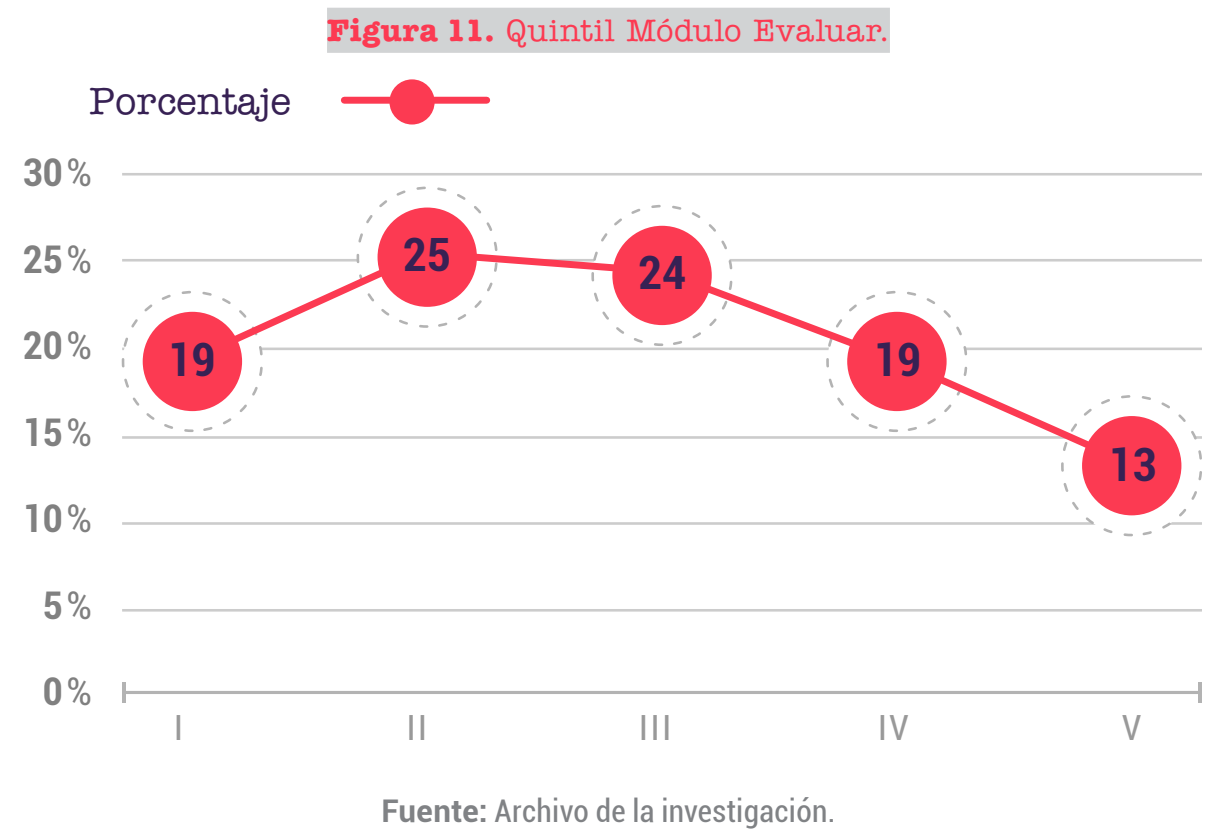

En la Figura 11 se observa una gran concentración de porcentaje de estudiantes en los quintiles I,II y III. De igual manera, en los quintiles IV y $\vee$ hay muy poco porcentaje de estudiantes agrupados allí, lo ideal es que los estudiantes concentrados en los primeros tres quintiles puedan pasar a hacer parte de los quintiles IV y $\vee$ y así poder mejorar en estos desempeños.

Tabla 16. Quintil Módulo Formar Año 2013.

\begin{tabular}{c|c|c|c|c} 
Quintil & Frecuencia & Porcentaje & Porcentaje válido & Porcentaje acumulado \\
\hline I & 52 & 19 & 19 & 19 \\
\hline II & 67 & 25 & 25 & 44 \\
\hline III & 66 & 24 & 24 & 68 \\
\hline IV & 53 & 19 & 19 & 87 \\
\hline V & 34 & 13 & 13 & $100 \%$ \\
\hline Total & $\mathbf{2 7 2}$ & $\mathbf{1 0 0} \%$ & & \\
\hline
\end{tabular}

Fuente: Archivo de la investigación.

En la Tabla 16 se observa la clasificación del porcentaje de estudiantes de acuerdo con el quintil dentro del grupo de referencia educación para el Módulo Evaluar. El 68\% de los estudiantes del programa 
de Licenciatura en Educación Básica con Énfasis en Matemáticas, Humanidades y Lengua Castellana hacen parte del $60 \%$ de los estudiantes con los menores puntajes y el $32 \%$ restante del $40 \%$ de los estudiantes con puntajes más altos.

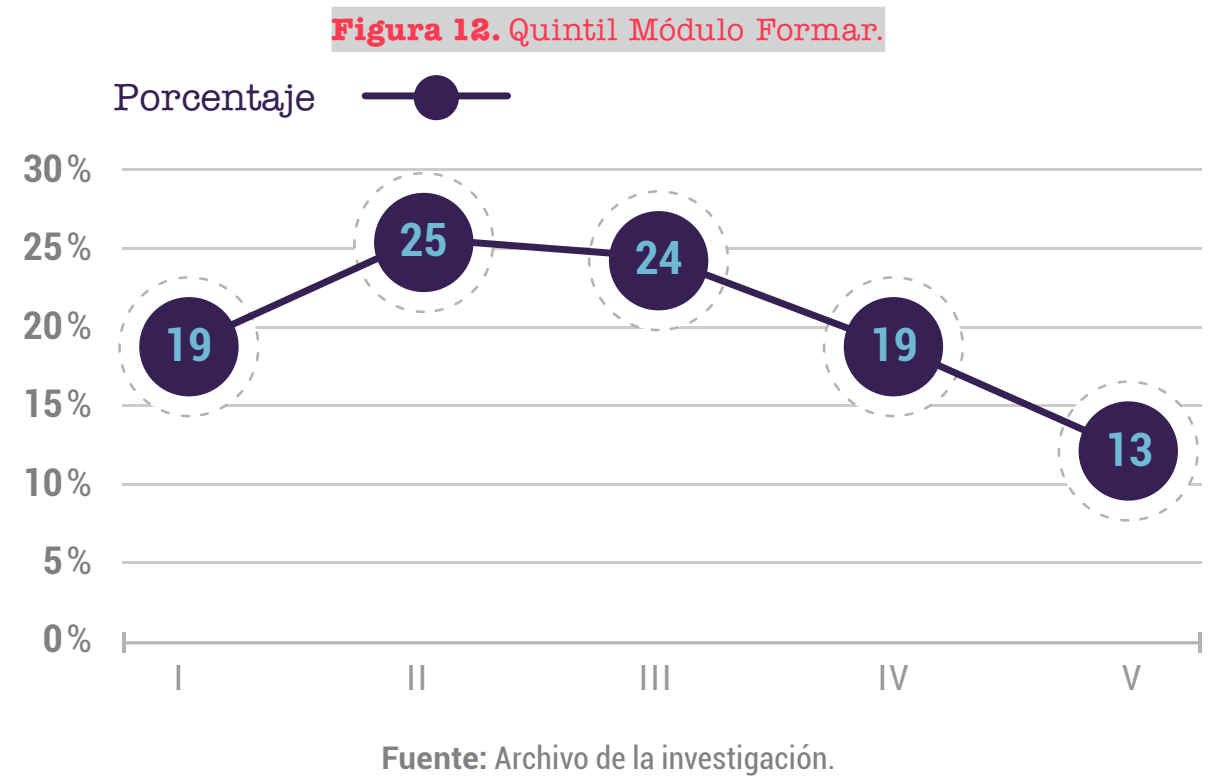

En la Figura 12 se observa una gran concentración de porcentaje de estudiantes en los quintiles I, II y III, presentándose así una disminución en los quintiles IV y $\mathrm{V}$.

El reto es concentrar la mayor catidad de estudiantes en los quintiles IV y V.

Tabla 17. Resultados módulos específicos Educación año 2013.

\begin{tabular}{c|c}
\hline COMPONENTE & PROMEDIO \\
\hline Enseñar & 9.93 \\
\hline Evaluar & 10.01 \\
\hline Formar & 9.9 \\
\hline
\end{tabular}

Fuente: Archivo de la investigación.

En la Tabla 17 se muestran los promedios obtenidos en los módulos del componente específico para el grupo de referencia educación. Se puede observar que los resultados obtenidos en los módulos de Enseñar y Formar se presenta una homogeneidad, en cuanto al módulo Evaluar presenta el promedio más alto con 10.01 y el de menor promedio el módulo Formar con 9.9. 
Figura 13. Análisis de los módulos específicos Educación año 2013.

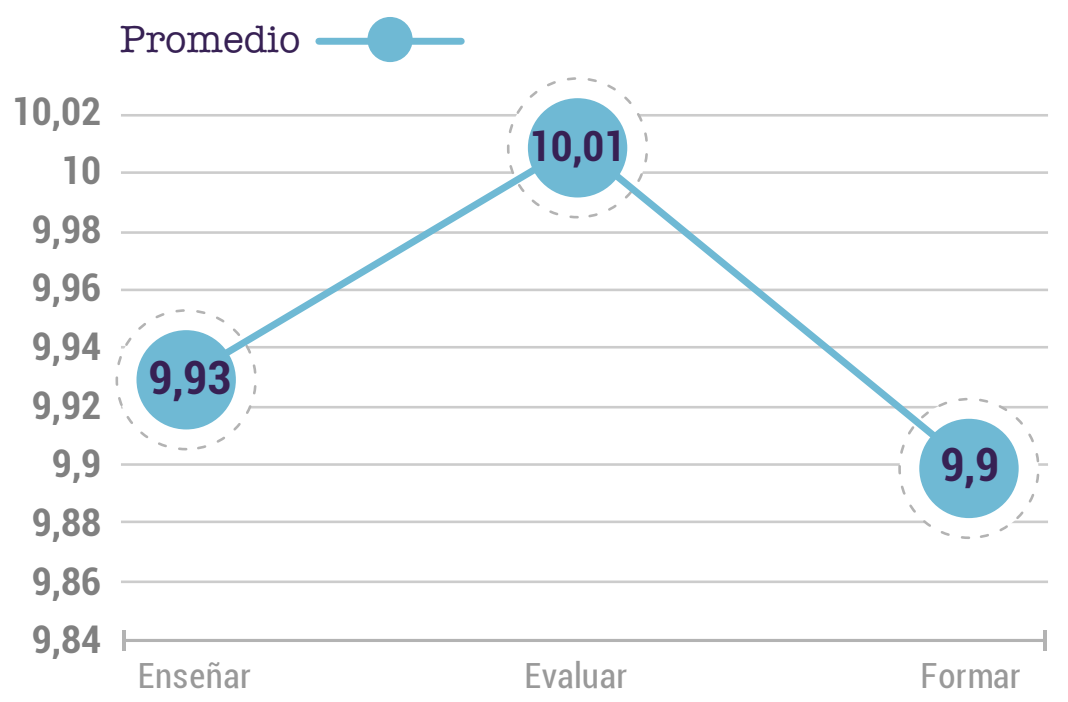

Fuente: Archivo de la investigación.

La Figura 13 muestra que el módulo con mejor promedio de desempeño es el de Evaluar con un puntaje de 10.01, este módulo evalúa competencias para hacer seguimiento, reflexionar y tomar decisiones en torno a los procesos de formación, con la intención de favorecer la autorregulación y plantear acciones de mejora en la enseñanza, en el aprendizaje y en el currículo relacionadas con la comprensión, la formulación y el uso de la didáctica de cada una de las disciplinas. En la figura se observa que el módulo con menor promedio es el de Formar con 9.9. Es importante tomar acciones para la mejora continua en los procesos de formación de licenciados.

\section{Tabla 19 Comparativo Programa, Grupo de Referencia}

y Nacional Pruebas Específicas

\begin{tabular}{c|c|c|c|c|c|c|c|c|c} 
Módulo & \multicolumn{2}{|c|}{ Programa } & \multicolumn{3}{c}{ Grupo de referencia } & \multicolumn{3}{c}{ Nacional } \\
\hline & N & Promedio & N & Promedio & Desviación & N & Promedio & Desviación \\
\hline Enseñar & 270 & 9.93 & 22439 & 10.1 & 1.1 & 183217 & 10.4 & 1 \\
\hline Evaluar & 270 & 10.01 & 22439 & 10.02 & 0.9 & 183217 & 10.5 & 1.2 \\
\hline Formar & 270 & 9.9 & 22439 & 10.3 & 1 & 183217 & 10.2 & 1 \\
\hline
\end{tabular}

Fuente: Archivo de la investigación.

En la Tabla 19 se puede apreciar el comparativo de los promedios del programa con relación al grupo de referencia y nacional en los módulos de competencias específicas del licenciado. También se observa en la tabla que los puntajes del programa varían de un mínimo de 9.9 
en el Módulo de Formar a un máximo de 10.01 en el Módulo de Evaluar. Asimismo, los del grupo de referencia varían de un mínimo de 10.02 en el Módulo de Evaluar a un máximo de 10.3 en el Módulo Formar. De igual forma el promedio nacional varía de un mínimo de 10.2 en el Módulo de Formar a un máximo de 10.5 en el Módulo de Evaluar.

Figura 14. Comparativo Programa, Grupo de Referencia

y Nacional Pruebas Específicas.

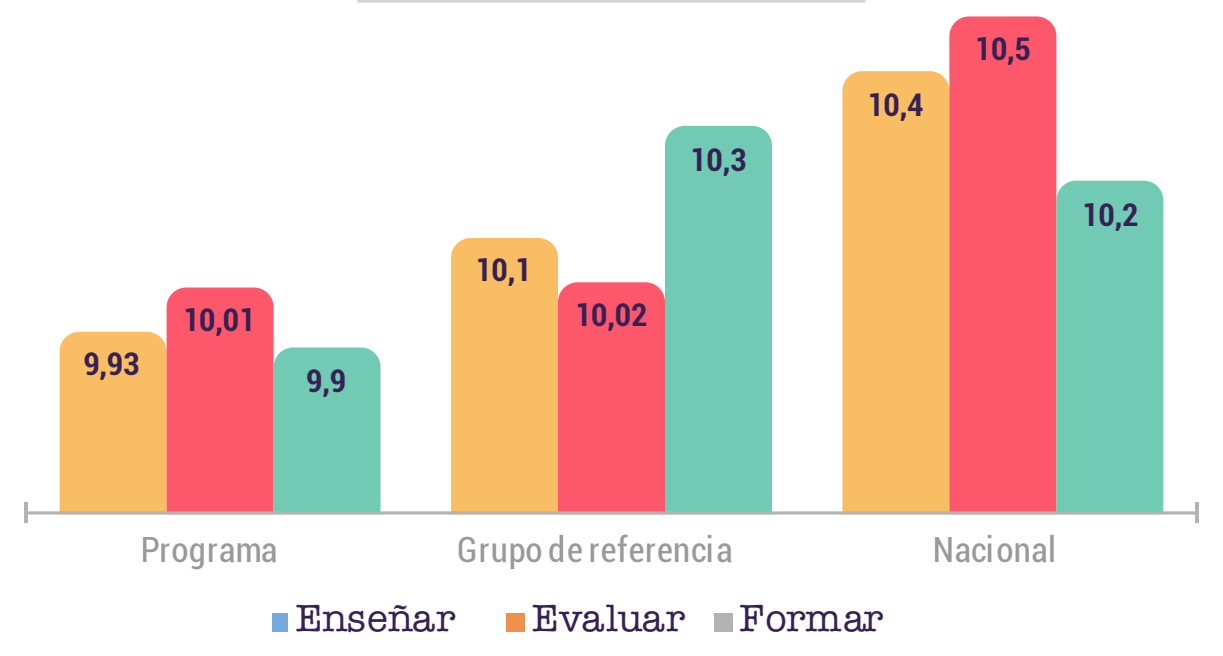

Fuente: Archivo de la investigación.

En la Figura 14 se aprecia el desempeño de los estudiantes del programa frente a los promedios del grupo de referencia y nacional en los módulos de competencias específicas del maestro. En el Módulo Enseñar el promedio del programa está por debajo tanto del promedio del grupo de referencia Educación y el promedio nacional. Con relación al Módulo Evaluar se observa que el promedio del programa está por encima del promedio del grupo de referencia Educación, pero por debajo del promedio nacional. En el Módulo Formar el promedio del programa se encuentra por debajo tanto del grupo de referencia Educación como del promedio nacional.

\section{Conclusiones}

A propósito, el campo de la calidad educativa es un escenario de debate, disertación y diálogo de saberes y se puede analizar en sentido amplio e incluso asociado con la cobertura. De manera 
que el compromiso de Egido (2005 y 2006) entre otros investigadores del objeto de estudio en debate continúa para avanzar la consolidación de un status quo de calidad, evaluación y calidad educativa. La importancia de la evaluación de la calidad educativa, los resultados de aprendizaje y la eficacia de los sistemas educativos son consecuencia de la articulación del proceso entre los actores de la educación para que se guarde coherencia sistemática entre calidad, evaluación y eficacia para la consecución de los resultados óptimos en la educación.

Los estudiantes de la Licenciatura en Educación Básica con enfasis en Matemáticas, Humanidades y Lengua Catellana que presentaron las pruebas Saber Pro en el año 2013 fueron objeto de evaluación homogénea y estándar en: Competencias Ciudadanas, Comunicación Escrita, Inglés, Lectura Crítica, Razonamiento Cuantitativo. En los resultados y análisis se puede observar que los promedios nacionales, grupo de referencia y del programa de los cinco módulos de competencias genéricas varían de un mínimo de 9.7 en el Módulo de Lectura Crítica a un máximo de 10.4 en el Módulo de Competencias Ciudadanas. Asimismo, los del grupo de referencia varían de un mínimo de 9.86 en el Módulo de Competencias Ciudadanas a un máximo de 10.4 en Razonamiento Cuantitativo. En relación al promedio nacional se encuentra entre un mínimo de 9.5 en el Módulo de Razonamiento Cuantitativo a un máximo de 9.9 en el Módulo de Comunicación Escrita.

Para los estudiantes de la Licenciatura en Educación Básica con enfasis en Matemáticas, Humanidades y Lengua Catellana en relación a las competencias genéricas, se evidencia que las Competencias Comunicaticas son las más altas con 10.4 y que la que ha logrado el nivel más bajo ha sido Lectura Crítica. Del grupo de referencia el de más alto procentaje es Razonamiento Cuantitativo de 10.4 y el de menor resultado son las Competencias Ciudadanas. Además, se evidencia un contraste en el caso de Competencias Ciudadanas, el más alto del programa de Licenciatura, pero el más bajo en relación al grupo de refrerencia. Y a nivel nacional el programa está por encima de los promedios de referencia, ubicando un desarrollo óptimo de los estudiantes que han presentado esta prueba estandarizada, que no tiene repercusión personal, pero sí es significativa para la medición de la Educación Superior en Colombia.

Para los estudiantes del programa, en relación al desempeño en los módulos de competencias específicas para un profesional en Educación: Enseñar, Evaluar y Formar se evidencia que los resultados varían de un mínimo de 9.9 en el Módulo de Formar a un máximo de 10.01 en el Módulo de Evaluar. Asimismo, los del grupo de referencia varían de un mínimo de 10.02 en el Módulo de Evaluar a un máximo de 10.3 en el Módulo Formar. De igual forma, el promedio nacional varía de un mínimo de 10.2 en el Módulo de Formar a un máximo de 10.5 en el Módulo de Evaluar.

A nivel del programa se mantiene un resultado similiar de los tres: Evaluar con 10.1, Enseñar con 9.93 y Formar 9.9. En el Módulo Enseñar el promedio del programa está por debajo tanto del promedio del grupo de referencia Educación 10.1 y el promedio nacional que es de 10.4. Con relación al Módulo Evaluar se observa que el promedio del programa está por encima del promedio del grupo de referencia Educación 10.02, pero por debajo del promedio nacional que es de 10.5. En el Módulo Formar el promedio del programa se encuentra por debajo tanto del grupo de referencia Educación 10.3 como del promedio nacional que es de 10.2; esto 
genera especial preocupación por los resultados de desempeño en los módulos específicos que ha de apropiar un licenciado y conlleva a futuro usar estrategias en donde se promueva una mejor preparación para presentar la prueba y como consecuencia mejores resultados.

\section{Referencias}

Ayala-García, J. (2015). Evaluación externa y calidad de la educación en Colombia. Documentos de Trabajo Sobre Economía Regional, (17). Banco de la República - Sucursal Cartagena. Recuperado de: http://banrep.gov.co/docum/Lectura_finanzas/pdf/dtser_217.pdf

Celis, M., Jiménez, O. \& Jaramillo J. (2006). ¿Cuál es la brecha de la calidad educativa en Colombia en la educación media y en la superior? Recuperado de: www.icfes.gov.co

Egido, I. (2005). Reflexiones en torno a la evaluación de la calidad educativa. Universidad Autónoma de Madrid. Revista Tendencias Pedagógicas, (10), 17-28. Recuperado de: https://repositorio. uam.es/handle/10486/4734

Egido, I. (2006). La evaluación de la calidad de la educación: entre la oportunidad y el riesgo. Revist a Educativa, 9 (1), 9-23.

Evaluación. (Enero-marzo 2006). Altablero. El periódico de un país que educa y que se educa. Recuperado de: https://www.mineducacion.gov. co/1621/propertyvalue-33074.html

Gómez, R. (2004). Calidad educativa: más que resultados en pruebas estandarizadas. Revista Educación y Pedagogía, 16(38), 75-89.

Guevara, R. (2017). La calidad, las competencias y las pruebas estandarizadas: una mirada desde los organismos internacionales. Revista Educación y Ciudad, (33). Recuperado de: https://bit. ly/2RXU4Hw
ICFES (2014). Acerca de las evaluaciones: ¿Cómo se elaboran las pruebas? Recuperado de: https:// bit.ly/2EsvfQZ

Ministerio de Educación Nacional (MEN). (2010). Sistemas de información. Recuperado de: https:// www.mineducacion.gov.co/1759/w3-channel. html?_noredirect=1

Ravela P., Arregui, P., Valverde, G., Wolfe, R., Ferrer, G., Martínez, F., Aylwin, M., \& Wolff, L. (2008) Las evaluaciones educativas que América Latina necesita. Documento nro. 40. Programa de Promoción de la Reforma Educativa en América Latina y el Caribe (PREAL). Santiago de Chile: Inter-American Dialogue. Recuperado de https:// bit.ly/2Eal6qu

\section{Lecturas sugeridas adicionales}

Cifuentes, J. (2013). La evaluación como mediación del proceso enseñanza-Aprendizaje. Revista Magistro, 7 (14). Recuperado de https://bit. ly/2KQ0yWh

Cifuentes, Medina, José E. (2014). Las prácticas evaluativas: una reflexión pertinente en la modalidad a distancia. Rastros Rostros, 16 (30), 19-33. doi: https://doi.org/10.16925/ra.v16i30.815

Cifuentes, J. E. y Camargo, A. L. (2015). Las prácticas evaluativas de los docentes del programa a distancia Tecnología en Regencia de Farmacia. Itinerario Educativo, (65), 37-97. Recuperado de http://revistas.usb.edu.co/index.php/Itinerario/ article/view/1703/1479

Manteca, E., Coordinador Editorial (2003). Hacia una política integral para la formación y el desarrollo profesional de los maestros de educación básica. México: Cuadernos de discusión 1.

Ortiz, A. (2015). Pedagogía y docencia universitaria: Hacia una didáctica de la educación superior. (Tomo I). (1era. Ed). Bogotá: Distribooks Editores. 